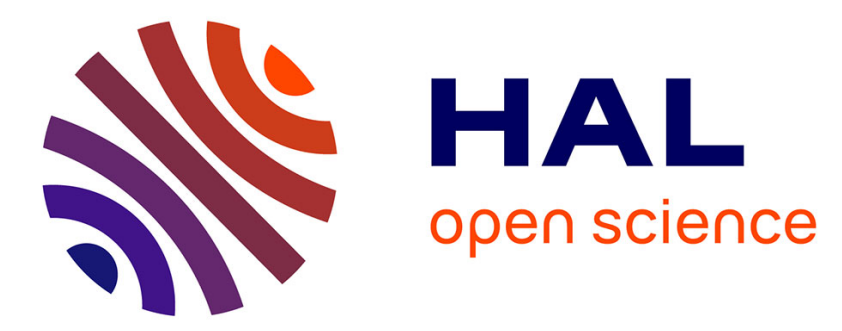

\title{
Entangled polymer chain solutions. Interpretation of the dipolar spin coupling modulation effect observed on NMR spectra
}

\author{
J.P. Cohen-Addad
}

\section{- To cite this version:}

J.P. Cohen-Addad. Entangled polymer chain solutions. Interpretation of the dipolar spin coupling modulation effect observed on NMR spectra. Journal de Physique Lettres, 1978, 39 (10), pp.147-150. 10.1051/jphyslet:019780039010014700 . jpa-00231464

\section{HAL Id: jpa-00231464 https://hal.science/jpa-00231464}

Submitted on 1 Jan 1978

HAL is a multi-disciplinary open access archive for the deposit and dissemination of scientific research documents, whether they are published or not. The documents may come from teaching and research institutions in France or abroad, or from public or private research centers.
L'archive ouverte pluridisciplinaire HAL, est destinée au dépôt et à la diffusion de documents scientifiques de niveau recherche, publiés ou non, émanant des établissements d'enseignement et de recherche français ou étrangers, des laboratoires publics ou privés. 


\title{
LE JOURNAL DE PHYSIQUE-LETTRES
}

Classification

Physics Abstracts

$61.40 \mathrm{~K}-76.60$

\section{ENTANGLED POLYMER CHAIN SOLUTIONS. INTERPRETATION OF THE DIPOLAR SPIN COUPLING MODULATION EFFECT OBSERVED ON NMR SPECTRA}

\author{
J. P. COHEN-ADDAD \\ Laboratoire de Spectrométrie Physique associé au CNRS, \\ Université Scientifique et Médicale de Grenoble, B.P. 53, 38041 Grenoble Cedex, France
}

(Reçu le 3 février 1978, révisé le 21 mars 1978, accepté le 29 mars 1978)

\begin{abstract}
Résumé. - Il est maintenant bien connu que les spectres RMN observés sur les solutions concentrées de chaînes de polymères enchevêtrées présentent un effet de rétrécissement induit par rotation de l'échantillon. Cependant, contrairement à ce qu'on attend d'une modulation du couplage dipolaire, aucune bande latérale n'est observable. Cette propriété est expliquée à partir de la réponse du système de spins calculée au quatrième ordre et en tenant compte de la modulation du couplage de spins. Une expression exacte du quatrième moment de l'ensemble du spectre est donnée. Il est montré que l'effet de cette modulation dépend de la forme de raie de résonance observée en l'absence de modulation; i) les bandes latérales doivent être bien observables lorsque la raie de résonance est gaussienne; ii) leur amplitude doit être négligeable lorsque la forme de la raie de résonance tend vers une lorentzienne.
\end{abstract}

\begin{abstract}
Concentrated entangled polymer chain solutions are known to exhibit a NMR spectrum narrowing effect upon sample rotation. However, the expected side bands normally induced by dipolar coupling modulation are not observed. To explain this property, the spin-system response to a driving field is calculated to fourth order in the dipolar spin interaction, taking the spin coupling modulation into consideration. The fourth moment of the whole spectrum is exactly expressed. It is shown that the spin interaction modulation effect depends upon the resonance line shape observed without modulation; i) the side bands should be well observable when the resonance line has a gaussian shape ; ii) the side band amplitudes become negligible when the resonance line has a nearly lorentzian shape.
\end{abstract}

1. Introduction. - A central question in the study of transverse nuclear magnetization dynamics observed on entangled polymer chain solutions is to prove that the average $\overrightarrow{\mathfrak{H}}_{\mathrm{D}}$ of the dipolar spin coupling established between all nuclei located on chains is non-zero : $\overline{\mathscr{H}}_{\mathrm{D}} \neq 0$. This problem has already led to the definition of the pseudosolid spin echo [1]. In this letter a detailed description of the observed dipolar spin coupling modulation effect is proposed. The non-zero average, $\overline{\mathfrak{H}}_{\mathrm{D}}$, necessarily implies that monomer units undergo non isotropic motions occurring on a time scale long enough compared with the decay time of the transverse nuclear magnetization. According to the most recent descriptions, it is considered that entangled polymer chains in semi-diluted solutions can form a statistical structure characterized by a correlation length, $\xi[2,3]$ and a disentanglement relaxation time $T_{\mathrm{r}}[4]$. Chain solutions as perceived from neutron scattering experiments performed on polystyrene can be pictured as divided into blobs [5] ; the average size, $v$, of a blob is determined from the space scale $\xi: v \propto \xi^{3}$; it contains $n \propto \xi^{5 / 3}$ monomer units. Excluded volume interactions dominate within a blob and chain segments behave like a free chain. The old entanglement concept [6] and the structure of solutions are connected with each other by identifying $\xi$ with the average distance between two consecutive chain coupling junctions or entanglements [4]. Properties observed on a molecular scale, $a(a \ll \xi)$ from low frequency proton magnetic relaxation processes reflect non-isotropic monomer unit motions. It is believed that these are induced by the presence of entanglements [7]. Using appropriate pulse sequences a pseudosolid spin echo can be observed on entangled polymer chains; its amplitude is proportional to a residual dipolar spin coupling.

Crucial evidence for a non-zero tensorial spin coupling average is provided by the observation of a 
spectrum narrowing effect induced by the spin interaction time modulation, at an appropriate angular frequency, $\Omega$. This well known effect has been widely applied to solids $[8,9]$. The rotation axis being perpendicular to the steady magnetic field direction, the linewidth, $\Delta$, is reduced by a factor of two when the sample is spun at frequencies higher than $\Delta$. At intermediate frequencies, spectra are expected to display side bands.

In studying NMR spectra from entangled polymer chains during sample rotation to modulate the dipolar spin coupling, one is faced with the two apparently conflicting observations [10] :

i) At high modulation frequency the linewidth is actually reduced by 1.8 or 2 .

ii) But no side bands can be observed whatever the modulation frequency; a careful experimental procedure shows that a side band amplitude equal to about $2 \%$ of the central resonance line amplitude exists. This surprising result raises questions regarding the residual dipolar spin coupling. However, in all cases it is noticed that the resonance line observed without modulation is nearly described by a lorentzian shape. Therefore, it seems that the absence of side bands may. well be associated with the lorentzian resonance line shape.

The aim of this letter is to explain the two observations.

2. The spin-system response. - No quantitative description theoretical or experimental of side bands has been given until recently [7] : side band amplitudes have now been calculated to second order in the spin interaction as a function of the modulation frequency $\Omega$; such a description is quite valid in the case where the line broadening mechanism leads to a gaussian resonance line shape; the second and fourth moments $M_{2}^{0}, M_{4}^{0}$ obey the relation $M_{4}^{0} \simeq 3 M_{2}^{0}$. In no cases can a lorentzian resonance line $\left(M_{4}^{0} \gg M_{2}^{0}\right)$ be described to second order in the spin interaction; therefore in the present letter, the spin-system response to a driving radiofrequency field is calculated to the fourth order, including dipolar coupling modulation. Because of the modulation, the spin interaction does not commute with itself at all times :

$$
\left[\overline{\mathscr{H}}_{\mathrm{D}}(t), \overline{\mathscr{H}}_{\mathrm{D}}\left(t^{\prime}\right)\right] \neq 0
$$

this is the only difficulty of such a calculation. A convenient technique is provided by the ordered exponential operator which applies to any operator $A$, according to the series expansion :

$$
\begin{aligned}
& R(t) A=U A+\sum_{p}(-i)^{p} \int_{0}^{t} \mathrm{~d} t_{1} \times \\
& \quad \times \int_{0}^{t_{1}} \mathrm{~d} t_{2}\left[\overline{\mathscr{H}}_{\mathrm{D}}\left(t_{1}\right),\left[\ldots\left[\overline{\mathscr{H}}_{\mathrm{D}}\left(t_{p}\right), A\right] \ldots\right]\right]
\end{aligned}
$$

$R(t)$ has the time ordering from the left; $R^{-1}(t)$ has the time ordering from the right and $i$ is replaced with $-i ; U$ is the unity matrix. The susceptibility is expressed as [7] :

$$
\begin{aligned}
V \chi^{\prime \prime}(\omega) & =\left(\omega_{0} / 2 k T \operatorname{Tr} U\right) \int_{0}^{\infty} S(u) \cos (\Delta \omega u) \mathrm{d} u \\
S(u) & =\operatorname{Tr}\left\{M_{x} R(t) R^{-1}(t-u) M_{x}\right\}
\end{aligned}
$$

$\Delta \omega=\omega-\omega_{0}, \omega_{0}$ is the central frequency, $V$ is the sample volume. $S(u)$ is now calculated to the fourth order in the spin interaction $\overline{\mathscr{H}}_{\mathrm{D}}$, the order generally used to describe lorentzian line shapes. A tedious but straightforward calculation shows that the fourth order term can be expressed as :

$$
\int_{0}^{u} \mathrm{~d} t_{1} \int_{0}^{t_{1}} \mathrm{~d} t_{2} \int_{0}^{t_{2}} \mathrm{~d} t_{3} \int_{0}^{t_{3}} \mathrm{~d} t_{4} h\left(t_{1}, t_{2}, t_{3}, t_{4}\right)
$$

with

$h=\operatorname{Tr}\left\{\left[\left[M_{x}, \overline{\mathscr{H}}_{\mathrm{D}}\left(t_{4}\right)\right], \overline{\mathscr{H}}_{\mathrm{D}}\left(t_{3}\right)\right]\left[\overline{\mathscr{H}}_{\mathrm{D}}\left(t_{2}\right),\left[\overline{\mathscr{H}}_{\mathrm{D}}\left(t_{1}\right), M_{x}\right]\right]\right\}$.

This simple expression results from the symmetry properties of $h$ and the following single hypothesis : the time variables $t_{p}(p=1,2,3,4)$ are supposed to be involved in the $h$ function through differences $t_{p}-t_{p^{\prime}}$, only. Now, this hypothesis is rigorously satisfied whenever the observed resonance line does not exhibit any angular dependence with respect to the steady magnetic field direction which is supposed to be perpendicular to the sample rotation axis. The structure of one of the typical time dependent terms involved in the trace operation (4) is :

$$
\sum_{i<j}\left(a_{i j}+b_{i j} \exp (2 i \Omega t)+\bar{b}_{i j} \exp (-2 i \Omega t)\right) A_{i j}
$$

$A_{i j}$ is the spin operator $\left(S_{i} S_{j}-3 S_{i z} S_{j z}\right)$;

$$
\begin{aligned}
& a_{i j}=-\alpha_{0} Y_{2}^{0}\left(\omega_{i j}\right) / 2 ; \\
& b_{i j}=\alpha_{0} \sqrt{3 / 8} Y_{2}^{-2}\left(\omega_{i j}\right) ; \\
& \alpha_{0}=\sqrt{4 \pi / 5} \gamma^{2} \hbar^{2} / r_{i j}^{3} .
\end{aligned}
$$

$Y_{2}^{m}(m=0, \pm 2)$ are spherical harmonics; $\omega_{i j} \equiv\left(\theta_{i j}\right.$, $\left.\varphi_{i j}\right)$ are the angular coordinates of the $\mathbf{r}_{i j}$ vector joining nuclei $i$ and $j$; these coordinates are defined in a frame associated with the sample; this frame is related to the laboratory frame through a rotation determined from Euler angles $\alpha=\Omega t, \beta=\pi / 2$ and $\gamma=0$. To examine the angular dependence of the fourth order term, the variable $\Omega t_{p}(p=1,2,3,4)$ is set equal to $\delta$. The angular invariance enables all terms which exhibit a $\delta$ dependence to be eliminated. A straightforward analysis shows that the only normalized terms consistent. with the angular invariance of the fourth order contribution are : $m_{4}^{0}, m_{4}^{1}$ 
and $m_{4}^{2}$ such that : $m_{4}^{0}+m_{4}^{1}+m_{4}^{2}=M_{4}^{0} ; M_{4}^{0}$ is the fourth moment of the resonance line calculated without any dipolar coupling modulation :

and

$$
m_{4}^{0}=\Sigma(n) a_{i j} a_{k l} a_{p q} a_{r s} A=M_{4}^{0} / 16
$$$$
m_{4}^{2}=4 \Sigma(n)\left|b_{i j}\right|\left|b_{k l}\right|\left|b_{p q}\right|\left|b_{r s}\right| A
$$

with $n \equiv(i<j, k<l, p<q, r<s)$ and

$$
A=\operatorname{Tr}\left\{\left[M_{x}, A_{r s}\right] A_{p q}, A_{k l},\left[A_{i j}, M_{x}\right]\right\} / \operatorname{Tr}\left\{M_{x}^{2}\right\}
$$

The angular invariance implies that $\delta$ is involved through products of conjugate exponentials only : $\exp ( \pm 2 i \delta)$; accordingly, only differences $t_{p}-t_{p^{\prime}}$ appear in the $h$ function.

3. Description of side bands. - The spin-system correlation function is written as :

$$
\begin{aligned}
G(u)= & S(u) / S(0) \simeq 1-m_{2}^{0} u^{2} / 2+ \\
& +m_{2}^{1}(\cos 2 \Omega u-1) / 4 \Omega^{2}+m_{4}^{0} u^{4} / 4 ! \\
& +m_{4}^{1} u^{2}(1-\cos 2 \Omega u) / 2 \Omega^{2}(4 !) \\
& +m_{4}^{2}(\cos 2 \Omega u-1)^{2} / 4 \Omega^{4}(4 !)+\cdots .
\end{aligned}
$$

Several features can be perceived from the series expansion (8). i) While the second moment $\left(-\mathrm{d}^{2} G / \mathrm{d} u^{2}\right)_{u=0}$ of the whole spectrum is invariant under rotation, as it should be, the fourth moment $\left(\mathrm{d}^{4} G / \mathrm{d} u^{4}\right)_{u=0}$ is a function of $\Omega$ :

$$
M_{4}(\Omega)=3 \Omega^{2} M_{2}^{0}+M_{4}^{0} .
$$

ii) It may be inferred that $G(u)$ is a function of $\sin ^{2} \Omega u / \Omega^{2}$ to all orders in the spin interaction. iii) When the modulation frequency $\Omega$ is very high, the only non-zero terms $1, M_{2}^{0} / 4, M_{4}^{0} / 16, \ldots$ lead to a resonance line broadening which is determined by $\overline{\mathscr{H}}_{\mathrm{D}} / 2$ instead of $\overline{\mathscr{H}}_{\mathrm{D}}$, whatever the exact line shape. To show that the whole spectrum may be pictured as a series of resonance lines at $\Delta \omega=0, \pm 2 p \Omega$, $\pm 4 p \Omega, . .$, the spin system correlation function is expressed as :

$$
\begin{aligned}
G(u)=g_{0}(u)+g_{1}(u) & \exp ( \pm 2 i \Omega u)+ \\
& +g_{2}(u) \exp ( \pm 4 i \Omega u)+\cdots
\end{aligned}
$$

with

$$
\begin{aligned}
& g_{0}(u)=\sigma_{0}(\Omega)-\mu_{2}^{0}(\Omega) u^{2} / 2+m_{4}^{0} u^{4} / 4 !+\cdots \\
& g_{1}(u)=\sigma_{1}(\Omega)-\mu_{2}^{1}(\Omega) u^{2} / 2+\cdots \quad \text { and } \\
& g_{2}(u)=\sigma_{2}(\Omega)+\cdots .
\end{aligned}
$$

The correlation functions $g_{0}(u), g_{1}(u)$ and $g_{2}(u)$ characterize the central line, the first and the second side bands, respectively. They are defined from series expansions :

$$
\begin{aligned}
& \sigma_{0}(\Omega)=1-m_{2}^{1} / 4 \Omega^{2}+m_{4}^{2} / 64 \Omega^{4} \\
& \sigma_{1}(\Omega)=m_{2}^{1} / 8 \Omega^{2}-m_{4}^{2} / 96 \Omega^{4} \quad \text { and } \\
& \sigma_{2}(\Omega)=m_{4}^{2} / 384 \Omega^{4} ;
\end{aligned}
$$

$\sigma_{q}(q=0,1,2)$ defines the relative area of each line. Also, the second moments are expressed as :

$\mu_{2}^{0}=m_{2}^{0}-m_{4}^{1} / 24 \Omega^{2}$ and $\mu_{2}^{1}=m_{4}^{1} / 48 \Omega^{2}$.

It is easily shown that the side band picture of the spectrum is quite in accordance with the exact relation :

$\mu_{2}^{0}+2 \mu_{2}^{1}+8 \Omega^{2} \sigma_{1}+32 \Omega^{2} \sigma_{2}=M_{2}^{0}$

and

$$
\begin{aligned}
m_{4}^{0}+32 \Omega^{4} \sigma_{1}+512 \Omega^{4} \sigma_{2}+ & 48 \Omega^{2} \mu_{2}^{1}= \\
& =M_{4}^{0}+3 M_{2}^{0} \Omega^{2}
\end{aligned}
$$

Explicit expressions for the different contributions to $M_{2}^{0}, m_{2}^{1}, M_{4}^{0}$ and $m_{4}^{2}$ terms can be determined from the trace properties of the spin operators and have been already calculated [11] :

$$
\begin{aligned}
& m_{2}^{0}= Q \sum_{j} a_{i j}^{2}=M_{2}^{0} / 4 ; Q=3 S(S+1) \\
& m_{2}^{1}= 3 M_{2}^{0} / 4=2 Q \sum_{j}\left|b_{i j}\right|^{2} \\
& m_{4}^{0}=Q^{2}\left\{3\left(\sum_{j} a_{i j}^{2}\right)^{2}-(3 N)^{-1} \times\right. \\
&\left.\quad \times \sum_{i \neq j \neq k} a_{i j}^{2}\left(a_{i k}-a_{j k}\right)^{2}-q \sum_{j} a_{i j}^{4}\right\}
\end{aligned}
$$

$q=1.6+0.3 / S(S+1) ; m_{4}^{2}$ has the same structure as $m_{4}^{0}: a_{i j}$ is only replaced with $\left|b_{i j}\right|$ and $Q^{2}$ with $4 Q^{2} ; m_{4}^{0}=M_{4}^{0} / 16$ and $m_{4}^{1}=M_{4}^{0}-m_{4}^{2}$.

4. Discussion. - The aim of the discussion is to characterize the dipolar spin coupling modulation effect from the resonance line shape. However it is well known that whenever the line shape is not a gaussian curve it is difficult to give a description with a simple analytical function. Accordingly, the modulation effect will be discussed considering an unusual but convenient parameter : the normalized first moment, $M_{1}$, calculated over half the NMR symmetrical spectrum

$$
M_{1}=\int_{0}^{\infty} \Delta \omega \chi^{\prime \prime}(\Delta \omega) \mathrm{d} \omega / \int_{0}^{\infty} \chi^{\prime \prime}(\Delta \omega) \mathrm{d} \omega
$$

When $G(u)$ is an even function, $M_{1}$ is simply expressed as :

$$
\begin{aligned}
& M_{1}=(\Pi)^{-1} \int_{-\infty}^{\infty} \varphi(u) \mathrm{d} u \\
& \varphi(u)=u^{-1} \mathrm{~d} G / \mathrm{d} u .
\end{aligned}
$$

The main feature which is perceived from formula (18) is that the first moment of the half spectrum is defined from an integral. This interesting property allows the use of an analytical representation of $\varphi(u)$ which is not necessarily accurate; it is assumed that the detailed form of $\varphi(u)$ does not critically govern 
the value of the integral (18). In the absence of any sample rotation :

$\varphi(u) \simeq-M_{2}^{0}+M_{4}^{0} u^{2} / 6 \simeq$

$$
\simeq-M_{2}^{0} \exp \left(-\varepsilon^{2} M_{2}^{0} u^{2} / 2\right)
$$

with

$$
\varepsilon^{2}=M_{4}^{0} / 3\left(M_{2}^{0}\right)^{2}
$$

and :

$$
M_{1}^{0} \simeq\left(2 M_{2}^{0} / \Pi \varepsilon^{2}\right)^{1 / 2} .
$$

This is not very different from the width, $\delta$, currently associated with a truncated lorentzian curve [12] : $\delta=1.31 M_{1}^{0}$. For a gaussian curve :

$$
M_{1}^{0}=\left(2 M_{2}^{0} / \Pi\right)^{1 / 2} \quad \text { as is expected } .
$$

During sample rotation, the first moment $M_{1}$ of a spectrum which consists of side bands,

$$
\chi^{\prime \prime}(\Delta \omega)=\sum_{p} \chi_{p}^{\prime \prime}(\Delta \omega-2 \Omega p),
$$

would normally be expressed as :

with

$$
M_{1}(\Omega)=\sum_{p}\left(\mu_{1}^{p}+2 \Omega p h_{1}^{p}\right)
$$

$$
\mu_{1}^{p}=\Pi^{-1} \int_{0}^{\infty} \mathrm{d} \omega(\Delta \omega-2 \Omega p) \chi_{p}^{\prime \prime} .
$$

Considering a side band $\chi_{p}^{\prime \prime}$ and assuming it has a constant area, it is easily seen that given its resonance frequency $2 p \Omega$ the narrower the line, the smaller $\mu_{1}^{p}$.

$$
h_{1}^{p}=\Pi^{-1} \int_{0}^{\infty} \mathrm{d} \omega \chi_{p}^{\prime \prime} .
$$

From formula (8), the function $\varphi(u)$ is expressed as :

$$
\varphi(u)=\psi(u)-2 \sum_{p=0}^{2} 2 p \Omega u^{-1} \sin (2 \Omega p u) g_{p}(u)
$$

$\psi(u)=-m_{2}^{0}+m_{4}^{0} u^{2} / 6+m_{4}^{1}(1-\cos 2 \Omega u) / 24 \Omega^{2}$.

An approximate series expansion is obtained by exponentiating $\psi(u)$ :

$$
\psi(u) \simeq k(u) \sum_{p} I_{p}(X) \exp (-X) \exp (2 i \Omega p u)
$$

$k(u)=m_{2}^{0} \exp \left(-\varepsilon^{2} M_{2}^{0} u^{2} / 8\right) ; I_{p}(p=0, \pm 1, \ldots)$ are modified Bessel functions; $X=m_{4}^{1} /\left(24 m_{2}^{0} \Omega^{2}\right)$. It is seen from (18), (24) and (25) that :

$$
h_{1}^{p}=\Pi^{-1} \int_{-\infty}^{\infty} u^{-1} g_{p}(u) \sin (2 \Omega p u) \mathrm{d} u .
$$

It is now assumed that from (18), (23) and (27) the approximate expression of $\mu_{1}^{p}$ is :

$$
\begin{aligned}
\mu_{1}^{p} \simeq\left(M_{2}^{0} / 2 \Pi \varepsilon^{2}\right)^{1 / 2} I_{p}(X) \times \\
\times \exp (-X) \exp \left(-8 p^{2} \Omega^{2} / \varepsilon^{2} M_{2}^{0}\right) .
\end{aligned}
$$

This expression is exact in the case of a gaussian line. Otherwise, it must of course be handled with care ; it applies to $p=0, \pm 1$, at the most ; numerical values calculated from it, must be considered as estimates of the $\mu_{1}^{p}$ coefficients, only. Two limiting cases will now be considered to illustrate properties of the central line and the first side band when the sample is spun at a frequency $\Omega \simeq M_{1}^{0} ; \mu_{1}^{0}$ and $\mu_{1}^{1}$ will be compared with one another.

i) In the absence of sample rotation the line shape is gaussian. Therefore, $\varepsilon^{2}=1, m_{4}^{1}=6 m_{2}^{0} m_{2}^{1}$ and $m_{4}^{2}=3\left(m_{2}^{1}\right)^{2} ; X=0.29$ and $\eta=8 \Omega^{2} / \varepsilon^{2} M_{2}^{0}=5$. $\sigma_{0}=0.77, \sigma_{1}=0.10$ and $\sigma_{2}=0.011 ; \mu_{1}^{1}\left(\Omega=M_{1}^{0}\right) /$ $\mu_{1}^{0}(\Omega)=10^{-3}$. The exact line width $2.36\left(M_{2}^{0}\right)^{1 / 2}$ of the gaussian first side band is smaller than

$$
2 \Omega=\left(8 M_{2}^{0} / \Pi\right)^{1 / 2} .
$$

This well resolved side band does not spread out much over the frequency range $\omega<0$ as is indicated by $\mu_{1}^{1} / \mu_{1}^{0}=10^{-3}$.

ii) In the absence of sample rotation the line shape is lorentzian; $\varepsilon^{2}$ is set equal to 2.2 to apply approximate formulae (11); $X=1.15$ and $\eta=2.31$ and $\mu_{1}^{1}\left(\Omega=M_{1}^{0}\right) / \mu_{1}^{0}(\Omega) \simeq 50 \times 10^{-3} . \sigma_{0}=0.77, \sigma_{1}=0.11$ and $\sigma_{2}=0.01$. The first side band must now spread out over a frequency range much larger than $2 \Omega$; its amplitude should be much weaker than in the gaussian case.

The above discussion shows that the absence of side bands normally induced from dipolar coupling modulation does not question the residual dipolar spin coupling established between all nuclei located on entangled polymer chains in solutions; it is related to the lorentzian nature of the resonance line.

\section{References}

[1] Cohen-Addad, J. P. and Vogin, R., Phys. Rev. Lett. 33 (1974) 940.

[2] Des Cloizeaux, J., J. Physique 36 (1975) 281.

[3] Daoud, M. and Jannink, G., J. Physique 37 (1976) 973.

[4] De Gennes, P. G., Macromolécules 9 (1976) 587.

[5] Daoud, M. et al., Macromolécules 8 (1975) 804.

[6] Busse, W. F., J. Phys. Chem. 36 (1932) 2862.

[7] Cohen-Addad, J. P., Domard, M. and Herz, J., J. Chem Phys. 68 (1978) 1194.
[8] ANDRew, E. R., The Narrowing of NMR Spectra in Solids in Progress in NMR Spectroscopy, Vol. 8, Ed. J. W. Emsley, J. Feeney and L. H. Sutcliff (Pergamon Press, Oxford) 1972.

[9] HaEberlen, U., High Resolution NMR in Solids (Academic Press, New York) 1976.

[10] Cohen-Addad, J. P. and Roby, C., J. Chem. Phys. 63 (1975) 3095.

[11] Van Vleck, J. H., Phys. Rev. 74 (1947) 1168.

[12] Abragam, A., Principles of Nuclear Magnetism (Oxford U.P. London) 1961 